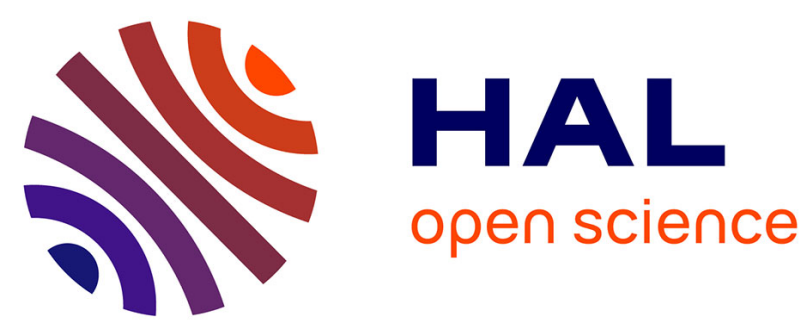

\title{
Symmetric Block-Matching Registration for the Distortion Correction of Echo-Planar Images
}

\author{
Renaud Hedouin, Olivier Commowick, Maxime Taquet, Elise Bannier, Benoit
} Scherrer, Simon K. Warfield, Christian Barillot

\section{- To cite this version:}

Renaud Hedouin, Olivier Commowick, Maxime Taquet, Elise Bannier, Benoit Scherrer, et al.. Symmetric Block-Matching Registration for the Distortion Correction of Echo-Planar Images. IEEE International Symposium on Biomedical Imaging (ISBI), Apr 2015, New York, United States. pp.717-720. inserm-01118766

\section{HAL Id: inserm-01118766 https://www.hal.inserm.fr/inserm-01118766}

Submitted on 6 May 2015

HAL is a multi-disciplinary open access archive for the deposit and dissemination of scientific research documents, whether they are published or not. The documents may come from teaching and research institutions in France or abroad, or from public or private research centers.
L'archive ouverte pluridisciplinaire HAL, est destinée au dépôt et à la diffusion de documents scientifiques de niveau recherche, publiés ou non, émanant des établissements d'enseignement et de recherche français ou étrangers, des laboratoires publics ou privés. 


\title{
SYMMETRIC BLOCK-MATCHING REGISTRATION FOR THE DISTORTION CORRECTION OF ECHO-PLANAR IMAGES
}

\author{
Renaud Hédouin ${ }^{\star} \quad$ Olivier Commowick ${ }^{\star} \quad$ Maxime Taquet $^{\dagger} \quad$ Elise Bannier $^{\star}$ \\ Benoit Scherrer $\quad$ Simon K. Warfield $\quad$ Christian Barillot ${ }^{\star}$ \\ * INRIA, INSERM, VisAGeS U746 Unit/Project, F-35042 Rennes, France \\ $\dagger$ Computational Radiology Laboratory, Children's Hospital Boston, USA
}

\begin{abstract}
We introduce a new approach to correct geometric and intensity distortion of Echo Planar Images (EPI) from images acquired with opposite phase encoding directions. A new symmetric block-matching registration algorithm has been developed for this purpose relying on new adapted transformations between blocks and a symmetric optimization scheme to ensure an opposite symmetric transformation. We present results of our algorithm showing its ability to robustly recover EPI distortion while obtaining sharper results than the popular TOPUP algorithm.
\end{abstract}

\section{INTRODUCTION}

Single-shot Echo Planar Imaging (EPI) is a very fast imaging method. Its low acquisition time allows us to acquire a large number of images in a reasonable time for a clinical usage. Diffusion Weighted Imaging (DWI) allows, through the acquisitions of many gradient weighted images with several bvalues, to model the underlying tissue microstructure of the brain. As many images are required to estimate water diffusion models, DWI needs many acquisitions. For this reason, EPI is the most common sequence used for DWI [1].

The major problem of EPI acquisitions resides in the large distortion that may affect the images. This distortion is caused by the magnetic field inhomogeneity at the tissue/air interfaces. Affected areas are contracted or dilated in the phase encoding direction (PED). To correct for this, several methods have been devised in the literature. One of the earliest techniques involves the acquisition of a $B_{0}$ field map [2], which is in turn used to estimate the $B_{0}$ inhomogeneities and correct for them. However, this method loses accuracy on strongly warped areas. Other techniques have considered the design of new sequences based on point spread functions [3] to obtain fast acquisitions with less distortion. Unfortunately such techniques, although very promising, are not available and ready to use in clinical routine on all scanners.

Another class of methods is based on the acquisition of an additional EPI where the PED is reversed. From those two images, a distortion field may be computed to correct the EPI. For example, Voss et al. [4] introduced a method to estimate from two images the correction displacement field based on cumulative intensity distributions along each line in the PED. Andersson et al. [5] followed the same kind of idea, which implementation is available in the popular FSL package (TOPUP). Other methods in this category include the one proposed by Holland et al. [6] performing a line intensity registration on each line independently. However, this method may be unstable when high noise or large displacements are present. Assuming that the subject did not move during a series of EPI acquisitions such as DWI, this class of algorithm allows us to correct for the distortion of the entire EPI series using only one supplemental $B_{0}$ image acquisition with reversed PED. Therefore, it allows for EPI distortion correction of DWI at almost no additional acquisition time cost.

We propose a new approach towards distortion correction of EPI based on a block-matching registration specifically designed to register two images acquired with opposite PEDs. To do so, we introduce a new symmetric block-matching registration algorithm, optimizing local affine transformations constrained in the PED for the expected distortions in EPI. In addition, the transformation is computed as opposite symmetric to match the distortion model in EPI [2]. We first present our distortion correction method in Section 2. Then, we apply it on real acquisition of five subjects where images with four different PED (anterior-posterior, posterioranterior, left-right, right-left) were acquired. We present results of our method in Section 4, demonstrating its ability to robustly recover EPI distortion while obtaining sharper results than TOPUP.

\section{METHODS}

\subsection{Distortion Model}

We assume that two images have been acquired: $I_{F}$ is the EPI acquired with a classical PED (e.g. anterior - posterior), and $I_{B}$ is the EPI acquired with a reversed PED (e.g. posterior anterior). The goal of EPI distortion correction is to recover a corrected image $C$ from these two images. Based on previous literature [2], we assume that $I_{F}$ and $I_{B}$ are generated from $C$ using a displacement field $U$ parallel to the PED:

$$
\begin{aligned}
& C(x)=J_{T_{+}}(x) I_{F}\left(T_{+}(x)\right) \\
& C(x)=J_{T_{-}}(x) I_{B}\left(T_{-}(x)\right)
\end{aligned}
$$

where $T_{+}(x)=x+U(x), T_{-}(x)=x-U(x), J$ denotes the Jacobian operator. $U$ corresponds to the distortion displacement field, it is assumed that $T_{+}$and $T_{-}$are opposite symmetric, i.e. that they share the same $U$ up to a minus sign. 


\subsection{Correction Initialization}

Distortion correction involves finding very large and local displacements between the images, displacements that may be extremely difficult to recover for registration approaches. We tackle this problem using a coarse-to-fine approach to recover EPI distortion. We have therefore used a coarse initialization to estimate an initial transformation, which is then further improved with our block-matching strategy. This initialization is based on the method introduced by Voss et al. [4]. Their approach amounts to the following. For each line in the PED, for both images do:

- Compute normalized cumulated intensities.

$$
N_{i}(y)=\frac{1}{\alpha_{i}} \int_{0}^{y} L_{i}(x) d x \quad \text { for } i=1,2
$$

where $L_{1}$ and $L_{2}$ are line intensities of images $I_{F}$ and $I_{B}, \alpha_{1}$ and $\alpha_{2}$ are normalization constants.

- For a large number $n$ of values $x_{n}$ between 0 and 1 , find by cubic interpolation $y_{1, n}$ and $y_{2, n}$ such that $N_{1}\left(y_{1, n}\right)=N_{2}\left(y_{2, n}\right)=x_{n}$

- At each position $y_{n}=\left(y_{1, n}+y_{2, n}\right) / 2$, the transformation map is computed as

$$
U\left(y_{n}\right)=y_{1, n}-y_{n}=y_{n}-y_{2, n}
$$

This algorithm has the advantage of being very fast and simple. However, it is highly sensitive to noise and lines registrations are purely independent which may lead to unrealistic transitions between lines. Therefore, Gaussian smoothing is performed on the obtained transformation $T$, which leads to a trade-off between transformation precision (small Gaussian $\sigma)$ and transformation regularity (high Gaussian $\sigma$ ).

\subsection{Block-Matching for Distortion Correction}

In the distortion model, the corrected image $C$ is unknown and can at best be estimated. We have therefore chosen a registration approach that does not rely on $C$. Avants et al. [7] have introduced a registration algorithm where, instead of looking for $T$ between two images, the transformation $T^{1 / 2}$ is sought so that $R \circ T^{-1 / 2}$ and $F \circ T^{1 / 2}$ match. We follow this idea, adapting it to a block-matching algorithm [8, 9] and to match the requirements of EPI distortion correction. Taking $U_{0}(x)=0$, the following steps are repeated at each iteration $l$ until convergence (for each pyramid level of a multi-resolution scheme):

- Resample: $I_{F, l-1}(x)=J_{T_{+, l-1}}(x) I_{F}\left(T_{+, l-1}(x)\right)$ and $I_{B, l-1}(x)=J_{T_{-, l-1}}(x) I_{B}\left(T_{-, l-1}(x)\right)$

- Estimate local transformations for each block on $I_{B, l-1}$ : $A_{+} \leftarrow$ block-matching $\left(I_{B, l-1}, I_{F, l-1}\right)$

- Estimate local transformations for each block on $I_{F, l-1}$ : $A_{-} \leftarrow \operatorname{block-matching}\left(I_{F, l-1}, I_{B, l-1}\right)$

- Extrapolate asymmetric dense stationary velocity field (SVF) corrections from $A_{+}$and $A_{-}$:

$\delta S_{+} \leftarrow \operatorname{extrapolate}\left(A_{+}\right), \delta S_{-} \leftarrow \operatorname{extrapolate}\left(A_{-}\right)$

- Compute symmetric SVF correction: $\delta S=\frac{1}{4}\left(\delta S_{+}-\right.$ $\left.\delta S_{-}\right)$, and compose it with current transformations: $T_{+, l}=T_{+, l-1} \circ \exp (\delta S)$ and $T_{-, l}=T_{-, l-1} \circ \exp (-\delta S)$
- Ensure $T_{+, l}$ and $T_{-, l}$ are opposite symmetric

- Regularize (elastic-like) $T_{+, l}$ and $T_{-, l}$

In the following, we will detail the block-matching, extrapolation and composition steps of this algorithm and their specificities for distortion correction of EPI.

\subsection{Block-Matching of Distorted EPI}

At each iteration, we define blocks $B_{+, i}$ regularly placed on image $I_{B, l-1}$, and $B_{-, i}$ on $I_{F, l-1}$. On each of those blocks, we look for an adapted transformation best matching $I_{B, l-1}$ and $I_{F, l-1}$. Such a transformation $A_{., i}$ can be constrained $a$ priori to match the expected features of distortion correction. First, $A_{., i}$ is defined as being collinear to the PED. Then, it should allow local contraction or dilation $\left(s_{i}\right)$ inside a block to account for local intensity changes in distorted images. Finally, we define skew components $k_{i}, m_{i}$ to the transformation so that it accounts for different translations parallel to the PED inside a block. Assuming the PED is the y-axis, $A_{., i}$ can be expressed as a $4 \times 4$ matrix of the following form:

$$
A_{., i}=\left(\begin{array}{cccc}
1 & 0 & 0 & 0 \\
k_{i} & s_{i} & m_{i} & t_{i} \\
0 & 0 & 1 & 0 \\
0 & 0 & 0 & 1
\end{array}\right)
$$

Such a transformation has several interesting properties: 1) its Jacobian is easily computed as the scaling component $s_{i}$ and can be used to approximate the local modification of intensities in the deformed images, 2) its matrix logarithm is analytically computed from its parameters which is important for the extrapolation step. With this transformation defined, we estimate $A_{+, i}$ and $A_{-, i}$ as the transformations (depending only on 4 parameters) optimizing a similarity measure $S$ between $I_{F, l-1}$ and $I_{B, l-1}$ respectively over the blocks $B_{+, i}$ and $B_{-, i}$ :

$$
\begin{aligned}
& \hat{A}_{+, i}=\underset{A_{+, i}}{\arg \max } S\left(J_{A_{+, i}} I_{F, l-1} \circ A_{+, i}, I_{B, l-1}\right) \\
& \hat{A}_{-, i}=\underset{A_{-, i}}{\arg \max } S\left(I_{F, l-1}, J_{A_{-, i}} I_{B, l-1} \circ A_{-, i}\right)
\end{aligned}
$$

A linear relationship can be assumed locally between the intensities of the blocks after correction and we therefore utilized a squared correlation coefficient as the similarity measure. This optimization is performed using the BOBYQA algorithm for bounded optimization without gradient [10]. This algorithm proceeds by successively computing quadratic approximations to the cost function to find its local maximum.

After this matching step, a confidence weight is assigned to each transformation. It is defined from two terms: a similarity based term, the optimal value of the similarity measure $\hat{S}$, and a directional weight $w_{d}$. The first term accounts for the quality of the best match. The second accounts for the image structure of the reference block. If the block image structure is parallel to the PED, registration in that direction is indeed undetermined, which is known as the aperture problem in image registration. We therefore define $w_{d}$ as a function of the structure tensor inside the reference block: $w_{d}(B(., i))=c_{l}\left(D_{B(., i)}\right)<\hat{v}_{D_{B(., i)}}, g>$, where $<., .>$ denotes the scalar product, $D_{B(., i)}$ the average structure tensor of block $B(., i), c_{l}$ is the linear coefficient [11], $\hat{v}_{D_{B(., i)}}$ 
is the principal eigenvector of $D_{B(., i)}$, and $g$ is the PED. This weight will be 0 if the structure tensor is perpendicular to the PED (image structure parallel to $g$ ) or spherical, giving a structure based confidence to the matches. In the end, the weight for the match of block $B(., i)$ is defined as $w_{B(., i)}=\sqrt{\hat{S}_{B(., i)} w_{d}(B(., i))}$.

\subsection{Transformation Extrapolation and Composition}

We have constituted two sets of block pairings (one for the $I_{F}$ and one for $\left.I_{B}\right): A_{+}=\left(A_{+, 1}, \ldots, A_{+, m}\right)$ and $A_{-}=$ $\left(A_{-, 1}, \ldots, A_{-, n}\right)$, each pairing being positioned at the center of its corresponding block, and given a weight $w_{B(., i)}$. We then use the method proposed in [9] to extrapolate two update SVFs from the sparse weighted log-transformations: $\delta S_{+}=$ extrapolate $\left(A_{+}\right)$and $\delta S_{-}=\operatorname{extrapolate}\left(A_{-}\right)$. A symmetric $\delta S$ is computed as a quarter of the subtraction of asymmetric incremental updates $\delta S_{+}$and $\delta S_{-}: \delta S=\frac{1}{4}\left(\delta S_{+}-\delta S_{-}\right)$, taking into account symmetry and the fact that only half of the SVF is required to make the images move towards the image $C$. This symmetrization is necessary to ensure equivalent roles to $I_{F}$ and $I_{B}$ in the registration process. Then, the transformation corrections $\delta T_{+}$and $\delta T_{-}$are computed as the exponential of these SVFs [12].

Such an extrapolation has the advantage of incorporating an outlier rejection framework, able to remove from the update SVF log-transformations that are erroneous due to noise or artifacts in the images. In addition, it guarantees that the extrapolated transformations are diffeomorphic and therefore invertible. Once $\delta T_{+}$and $\delta T_{-}$are computed, they are composed with the current transformations: $T_{+, l}=T_{+, l-1} \circ \delta T_{+}$ and $T_{-, l}=T_{-, l-1} \circ \delta T_{-}$. Such an operation however does not guarantee that $T_{+, l}$ and $T_{-, l}$ still have opposite roles, as required in the EPI distortion model, i.e. that $T_{+, l}(x)=$ $x+U_{l}(x), T_{-, l}(x)=x-U_{l}(x)$. We therefore compute $U$ at the $l-t h$ iteration as $U_{l}(x)=\frac{1}{2}\left(T_{+, l}(x)-T_{-, l}(x)\right)$, thereby ensuring an opposite symmetric transformation.

\section{EXPERIMENTAL DESIGN}

We have scanned 5 healthy volunteers to evaluate our distortion correction algorithm. For each volunteer, two pairs of $B_{0}$ EPI with opposite PED were acquired: AP/PA and LR/RL. Each image has a size of $128 \times 128 \times 60$ and an isotropic resolution of $2 \times 2 \times 2 \mathrm{~mm}^{3}$. We then corrected the AP/PA and the LR/RL images separately using one of the algorithms to evaluate: initial transformation as described in Section 2.2 (Initial), our algorithm initialized by the previous transformation (BM), and the TOPUP algorithm [5]. The two pairs of images $\mathrm{AP} / \mathrm{PA}$ and $\mathrm{LR} / \mathrm{RL}$ are subject to different distortion but are images of the same anatomy. We therefore measure the quality of an algorithm as a comparison between the two images after distortion correction.

To compare images after correction, we define two complementary measures. First, we specify a global measure Sim on images as an average of the local correlation coefficients between the images. However, intensities out of the brain are pure noise. We therefore masked those background areas by first computing a mask of the relevant areas for each corrected image. Then, a global mask was computed as the union of all these individual masks to ensure that quality metrics are comparable between the methods. Sim is then defined as:

$$
\operatorname{Sim}(I, J)=\frac{1}{\operatorname{Card}\left(\{I, J\}^{\mathcal{N}}\right)} \sum_{p \in\{I, J\}^{\mathcal{N}}} C\left(I_{\mathcal{N}_{p}}, J_{\mathcal{N}_{p}}\right)
$$

where $C$ is the local correlation of $I_{\mathcal{N}_{p}}, J_{\mathcal{N}_{p}}$ (neighborhoods of $p$ in $I$ and $J),\{I, J\}^{\mathcal{N}}$ the image domain. Sim characterizes well if the images match after correction, however it is sensitive to image smoothing which may make a method appear better than it really is. In fact, the resulting correction also needs to keep the structural information: the sharpness of the images. This is evaluated by a sharpness index as follows:

$$
\operatorname{Sharpness}(I)=\frac{1}{\operatorname{Card}\left(I^{\mathcal{N}}\right)} \sum_{p \in I^{\mathcal{M}}} \frac{V\left(I_{\mathcal{N}_{p}}\right)}{E^{2}\left(I_{\mathcal{N}_{p}}\right)}
$$

where $V$ is the variance and $E$ the expectation. It computes the average of ratios between the local variances and means. In other words, it considers that structures are sharply defined if the variance inside a block is high compared to its mean. Again, we compute this measure on the same mask used for correlation.

\section{RESULTS}

We present in Fig. 1 a representative example of a distortion correction result for $\mathrm{LR} / \mathrm{RL}$ images. This figure demonstrates visually that our algorithm clearly improves results over the initialization method. The eyes are better defined after applying BM, and structures in the temporal lobes are also better defined and more in phase with the anatomical T2 reference. TOPUP also achieves a good result, however it is more blurry than the other two methods.

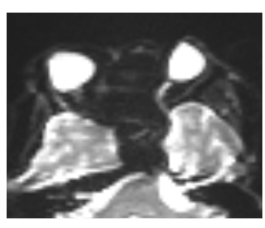

(a) LR

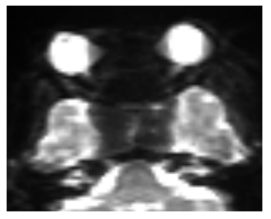

(d) Initial Correction

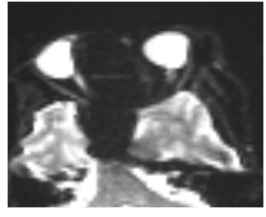

(b) RL

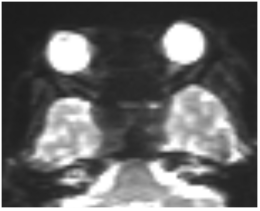

(e) BM

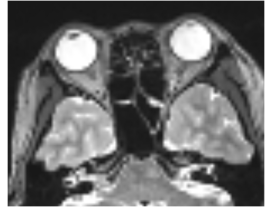

(c) $\mathrm{T} 2$

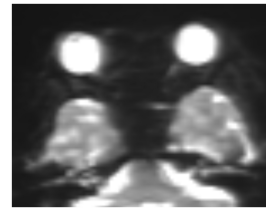

(f) TOPUP
Fig. 1. Distortion correction results. First line: EPI with opposite PED (LR/RL) and a T2 resampled image. Second line: correction results with the three compared methods.

To quantitatively validate our observations, we have computed similarity and sharpness metrics to our datasets. Correlation similarity results are presented in Table 1 . This table shows that BM increases systematically the similarity index as compared to the initialization. However, these results also show that TOPUP seems to be performing better than our method that could be due to a visually smoother result. 


\begin{tabular}{|c|c|c|c|c|}
\hline Sim & Untouched & Initial & BM & TOPUP \\
\hline Subject 1 & 0.50 & 0.56 & 0.65 & 0.76 \\
\hline Subject 2 & 0.48 & 0.53 & 0.59 & 0.71 \\
\hline Subject 3 & 0.51 & 0.56 & 0.62 & 0.64 \\
\hline Subject 4 & 0.62 & 0.68 & 0.72 & 0.81 \\
\hline Subject 5 & 0.64 & 0.68 & 0.72 & 0.80 \\
\hline
\end{tabular}

Table 1. Correlation results (Sim measure) between AP/PA and LR/RL images. Columns from left to right: no correction, initial transformation, BM and TOPUP.

To better understand the behavior of TOPUP, we therefore computed the sharpness on all images, corrected or not, on the 5 subjects. Results are presented in Table 2 with images and correction of the pair AP/PA, (results for the pair $\mathrm{LR} / \mathrm{RL}$ are similar). The first three columns (original images, corrected by initial transformation and by BM) show a stable sharpness index. However, the sharpness index of TOPUP decreases significantly, confirming the visual impression that TOPUP produces smoother images after correction.

\begin{tabular}{|c|c|c|c|c|}
\hline Sharpness & Untouched & Initial & BM & TOPUP \\
\hline Subject 1 & 0.34 & 0.33 & 0.36 & 0.23 \\
\hline Subject 2 & 0.38 & 0.36 & 0.39 & 0.26 \\
\hline Subject 3 & 0.35 & 0.33 & 0.36 & 0.25 \\
\hline Subject 4 & 0.32 & 0.31 & 0.33 & 0.23 \\
\hline Subject 5 & 0.34 & 0.33 & 0.34 & 0.24 \\
\hline
\end{tabular}

Table 2. Sharpness results of corrected images. From left to right: no correction, initial transformation, BM and TOPUP.

As a final comparison, we then tested the incidence of smoothness on the correlation score. We present in Table 3 the correlation results for one representative correction result obtained by BM, simply adding Gaussian smoothing to the result images with different $\sigma$ before computing the local correlations. Interestingly, correlation and sharpness are strongly linked: when increasing smoothness, the correlation score reaches better values. Moreover, for a specific $\sigma$ value (0.6), we even obtain the same sharpness and similarity values as for TOPUP. This advocates two conclusions : 1) our method obtains results of similar quality to TOPUP but with a higher sharpness of structures, which is desirable to avoid partial volume effects; 2) validation of distortion correction methods is a complex field that requires several metrics to be considered for evaluating all desired aspects.

\begin{tabular}{|c|c|c|c|c|c|c|}
\hline Sigma & 0.2 & 0.3 & 0.4 & 0.5 & $\mathbf{0 . 6}$ & 0.7 \\
\hline Sharpness & 0.36 & 0.35 & 0.32 & 0.27 & $\mathbf{0 . 2 3}$ & 0.21 \\
\hline Sim & 0.65 & 0.65 & 0.67 & 0.72 & $\mathbf{0 . 7 6}$ & 0.79 \\
\hline
\end{tabular}

Table 3. Illustration of the link between image smoothness and correlation results. First line: $\sigma$ values for Gaussian smoothing, second and third line: sharpness and correlation of BM corrected images after smoothing.

\section{CONCLUSION}

We have developed a new algorithm for the correction of EPI distortion using two EPI with opposite PED. To do so, we have developed new linear transformations adapted to local distortions. We then integrated these transformations into a symmetric block-matching algorithm, ensuring a robust computation of an opposite symmetric transformation. We have evaluated this method on 5 subjects with 2 pairs of EPI. We have shown how the block-matching approach improves quantitative and visual results. It also provides results comparable with TOPUP but with a much improved sharpness, close to the one of the original images. Computationally, we have run the two algorithms on a Xeon $2.5 \mathrm{Ghz}$ computer on 20 cores: our method took $150 \mathrm{~s}$ and TOPUP $750 \mathrm{~s}$.

\section{REFERENCES}

[1] H. Johansen-Berg and T.E.J. Behrens, Diffusion MRI: From quantitative measurement to in-vivo neuroanatomy, Academic Press, 2009.

[2] P. Jezzard and R. S. Balaban, "Correction for geometric distortion in echo planar images from B0 field variations.," MRM, vol. 34, no. 1, pp. 65-73, 1995.

[3] M. Zaitsev, J. Hennig, and O. Speck, "Point spread function mapping with parallel imaging techniques and high acceleration factors: Fast, robust, and flexible method for echo-planar imaging distortion correction," MRM, vol. 52, no. 5, pp. 1156-1166, 2004.

[4] H.U. Voss, R. Watts, A.M. Ulugc, and D. Ballona, "Fiber tracking in the cervical spine and inferior brain regions with reversed gradient diffusion tensor imaging," MRM, vol. 24, no. 3, pp. 231-239, 2006.

[5] J.L.R. Andersson, S. Skare, and J. Ashburner, "How to correct susceptibility distortions in spin-echo echo-planar images: application to diffusion tensor imaging," $\mathrm{Neu}$ roimage, vol. 20, no. 2, pp. 870-888, 2003.

[6] D. Holland, J.M. Kuperman, and A.M. Dale, "Efficient correction of inhomogeneous static magnetic fieldinduced distortion in echo planar imaging," Neuroimage, vol. 50, no. 1, pp. 175-183, 2010.

[7] B.B. Avants, C.L. Epstein, et al., "Symmetric diffeomorphic image registration with cross-correlation: evaluating automated labeling of elderly and neurodegenerative brain," MedIA, vol. 12, no. 1, pp. 26-41, 2008.

[8] S. Ourselin, A. Roche, S. Prima, and N. Ayache, "Block matching: A general framework to improve robustness of rigid registration of medical images," in MICCAI, 2000, vol. 1935 of $L N C S$, pp. 557-566.

[9] O. Commowick, N. Wiest-Daesslé, and S. Prima, "Automated diffeomorphic registration of anatomical structures with rigid parts: application to dynamic cervical MRI.," in MICCAI, 2012, vol. 15 of LNCS, pp. 163-70.

[10] M.J.D. Powell, "The BOBYQA algorithm for bound constrained optimization without derivatives," Tech. Rep., Centre for Mathematical Sciences, University of Cambridge, UK, 2009.

[11] C.-F. Westin, S.E. Maier, et al., "Processing and visualization for diffusion tensor MRI," MedIA, vol. 6, no. 2, pp. 93-108, 2002.

[12] V. Arsigny, O. Commowick, et al., "A fast and logeuclidean polyaffine framework for locally linear registration," JMIV, vol. 33, no. 2, pp. 222-238, Jan. 2009. 